\title{
Aparcamiento regulado a lo largo del parterre central de la vía: estudio de caso en la Avenida Recanto das Emas-DF
}

\author{
Hudson Carrer Pereira \\ Daliana Bandeira Luz Monteiro Santos \\ Erwin Joffre Delgado Bravo \\ PhD Student, Universidad de Coimbra, Portugal
}

\section{RESUMEN}

Este trabajo estudia el impacto en la seguridad vial de la aplicación en 2007 de plazas de aparcamiento a lo largo del parterre central de la Avenida Recanto das Emas - Distrito Federal, Brasil. El análisis se basó en la legislación brasileña, manuales técnicos y datos obtenidos de la Gerencia de Estadística del Departamento del Distrito Federal (GerestDetran/DF) sobre los tipos de accidentes y las cantidades en el período de tráfico antes y después de la construcción del aparcamiento a lo largo del parterre central. La ausencia de una separación física obligatoria y el paso de peatones a lo largo de la pista son algunos de los factores que contribuyeron al aumento del número de accidentes. La interferencia con la red de ciclismo y la política de aparcamiento también se pone en relieve en la planificación de la movilidad en favor de la sostenibilidad de la ciudad.

\section{INTRODUCCIÓN}

\subsection{Justificación y Objetivo del Trabajo}

El estudio de la Avenida Recanto das Emas, enfoque de este trabajo, fue motivado como apoyo a la evaluación de revitalización de avenida con características funcionales, geométrica y jerárquicamente similar (Av. Paranoá), donde una de las propuestas con fuerte atractivo era la que tenia el número más alto de los espacios de aparcamiento, dispuestos a lo largo del parterre central.

La elección de la Avenida Recanto das Emas fue dada por las semejanzas antes mencionadas, pero sobre todo porque en 2007 se construyeron aparcamientos a lo largo del parterre central. El impacto de la construcción de este aparcamiento en la seguridad vial es el objeto de trabajo. Es de destacar que en ambas avenidas hay ciclo vías construidas en el parterre central en 2011.

\subsection{Avenida Recanto das Emas}

Vía arterial con cerca de $8 \mathrm{~km}$ de largo, es la avenida principal de la región administrativa de Recanto das Emas, cuya población en 2015 se estimó en 145.304 habitantes, de acuerdo con la Companhia de Planejamento do Distrito Federal (2015). Un extremo de la carretera conecta con la Carretera BR 251, acceso para aquellos que deseen viajar a Brasilia y otras 
regiones.

El perfil de la vía cambia bastante a lo largo de su longitud, y la acera presenta comúnmente piso dañado, falta de accesibilidad, discontinuidades, desviaciones y variaciones de ancho con obstáculos por equipos móviles y fijos que ponen en peligro la libre circulación de los peatones. La pista de rodadura tiene cuatro carriles de 3,5 m de ancho cada uno, dos para cada dirección. Existen compartimentos de embarque y desembarque de los pasajeros del autobús. La velocidad regulada es de $60 \mathrm{~km} / \mathrm{h}$, con dos ubicaciones en la instalación de barreras electrónicas con reducción de velocidad a $50 \mathrm{~km} / \mathrm{h}$.

Un cambio significativo observado en la avenida, además de la construcción en 2007 del aparcamiento (el tema de este estudio), es que se construyó en 2011 una ciclo vía bidireccional con 2,2 $\mathrm{m}$ de ancho, también en el parterre central. El parterre central tiene una anchura aproximada de 12,5 m, y en una de las secciones-tipo la acera es de 2,0 m, ciclo vía con 2,2 m y césped. Donde fueron construidos aparcamientos de $5 \mathrm{~m}$ para maniobrar el vehículo, sin un divisor físico como la legislación lo establece. Además, en estos puntos la ciclo vía se interrumpe, dejando a los ciclistas de todas las edades que viajan en ambas direcciones en conflicto con vehículos que viajan en el carril izquierdo (mayor velocidad) y que quieren entrar en el aparcamiento. Las figuras la y $1 \mathrm{~b}$ ilustran la situación mencionada en el inicio (el bloqueo de la ruta de bicicleta por un vehículo) y en el final (ciclista en la dirección opuesta al flujo) de una de las secciones de aparcamiento.

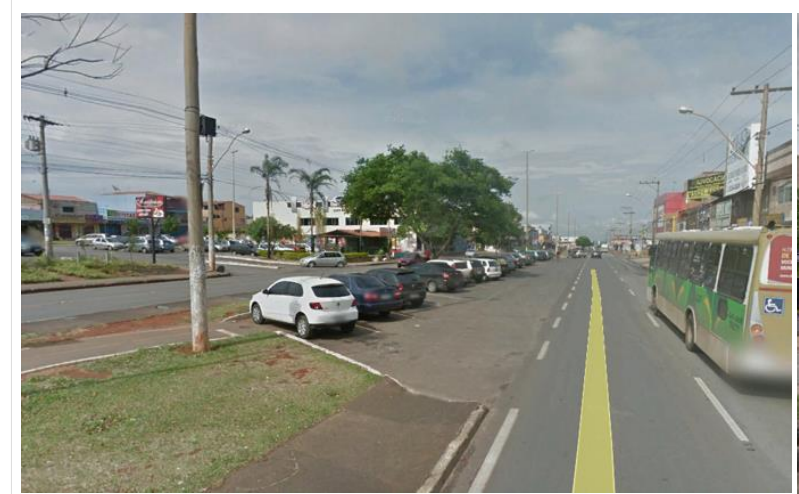

Fig. 1a - Inicio del aparcamiento

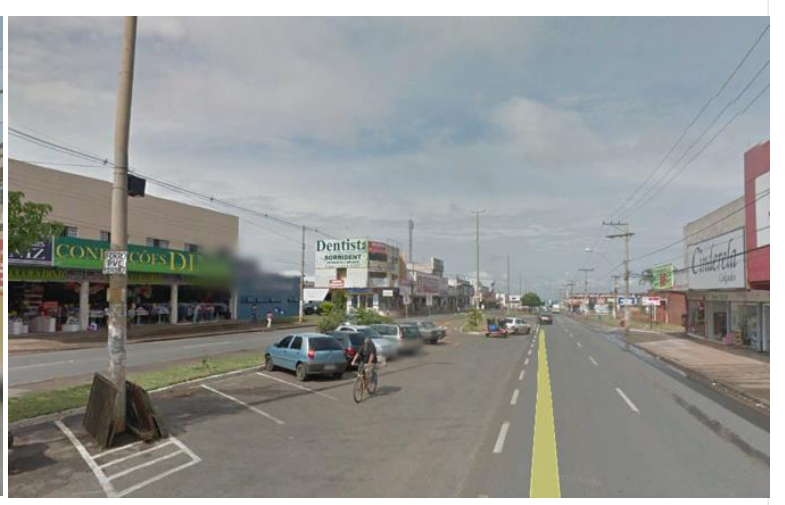

Fig. 1b Final del aparcamiento

\section{REVISIÓN DE LA LITERATURA}

\subsection{Legislación}

El Código de Tránsito Brasileño (CTB), establecido por la Ley Federal no 9.503/1997, define el parterre central como "barrera física construida como pestaña de dos carriles, opcionalmente sustituido por marcas viales (parterre ficticio).” El CTB tiene en el Art. 181, inciso VIII, que es infracción "aparcar el vehículo en la acera o en la pista destinada a los peatones, en el carril bici o ciclo vía, así como en las islas, refugios, junto o los parterres centrales, separadores de carril, marcas de canalización, césped o jardín público “. Para ser admitido el aparcamiento en estas plazas deben existir señales en condiciones específicas 
para este fin.

El Manual de Señalización Vertical del Reglamento (Contran, 2007) aclara que el signo de Aparcamiento Regulado (R-6b) se utiliza para permitir el aparcamiento en lugares que tienen, como regla general, la prohibición de aparcamiento y/o parada. El parterre central hace parte de los ejemplos de aplicación señal.

La Avenida Recanto das Emas es una vía arterial, y el CTB define vía arterial como "aquella caracterizada por intersecciones en nivel, generalmente controlada por semáforos, con la accesibilidad a las porciones limítrofes y a las vías secundarias y locales, permitiendo el tráfico entre las regiones de la ciudad". Esta clasificación jerárquica tiene un fuerte elemento de movilidad, aunque con consideraciones de accesibilidad.

El Distrito Decreto n ${ }^{\circ} 33.741 / 2012$, que regula el Art. 20 de la Ley Complementaria $n^{\circ}$ 803, de 25 de abril de 2009, en relación con las normas de circulación, conceptos generales y los parámetros para el dimensionamiento del sistema vial urbano para la planificación, preparación y modificación de proyectos urbanos, el Art. 36, Inciso III, Párrafo 1 establece que:

Art. 36. La creación de zona de aparcamiento público es permitida en los siguientes casos:

(...)

III - a lo largo de las vías.

$\S 1^{\circ}$ Para el estacionamiento a lo largo de las vías de tránsito rápido y las vías arteriales deben ser previsto divisor físico de circulación con al menos un metro de ancho.

Con base en lo anterior, se puede considerar que el parterre central es una ubicación inusual, pero permitida para las plazas de aparcamiento de vehículos señalizadas, y no hay ninguna mención de este permiso en relación con la jerarquía de carretera. Es de destacar que la Avenida Recanto das Emas no tiene divisor físico de circulación.

Con respecto a las normas de circulación de vehículos, Art. 29, Párrafo IV del CTB establece que "cuando una pista tiene varios carriles en el mismo sentido, las de la derecha sin destinadas a los vehículos más lentos y más grandes, cuando no hay pista especial destinada a ellos, y las de la izquierda son destinadas para el paso y el desplazamiento de los vehículos de mayor velocidad".

\subsection{Manuales Técnicos}

Según el Volumen 4 del Manual de Planeamento das Acessibilidades e da Gestão Viária (2008), las vías arteriales (llamadas en Portugal de distribuidoras principales) deben ser diseñadas y manejadas para asegurar un buen nivel de seguridad y niveles al menos razonables de fluidez y velocidad del flujo motorizado, con adopción de soluciones 
geométricas para evitar la congestión y permitir la circulación a velocidades adecuadas. Con respecto al aparcamiento, el manual esclarece que normalmente es permitido y se deben, casi siempre, realizar a lo largo y lejos de las intersecciones, sin hacer referencias específicas a lo parterre central para este fin.

De acuerdo con la Empresa de Transportes e Transito de Belo Horizonte - BHTRANS (2010), además la clasificación formal y las características operativas de la vía, la regulación de aparcamiento debe considerar el uso del suelo, el tráfico, las condiciones topográficas y la visibilidad, etc. En cuanto a la circulación de vehículos, la ubicación de aparcamiento a lo largo del parterre central implica un conflicto de aceleración/deceleración del vehículo en el carril izquierdo, previsto para los adelantamientos y el desplazamiento de los vehículos de mayor velocidad.

El uso del parterre central como aparcamiento también se refleja en el movimiento de los peatones. Los conductores que aparcan en la avenida realizan travesías no siempre por pasaderas y el fracaso de la canalización de los peatones en una vía arterial aumenta el riesgo de accidentes.

Específicamente en el caso de la Avenida Recanto das Emas se observa que la ciclo vía implantada en 2011, cuatro años después de la construcción del aparcamiento, tiene graves problemas de discontinuidad, obligando a los ciclistas, que se desplazan en ambos sentidos de circulación, a compartir el espacio vial con vehículo de motor en sentidos contrarios. El diseño de la ciclo vía termina frente a un coche, lo que implica bajar de la bicicleta para continuar la ruta, lo que dificulta la movilidad de los no motorizados. Por cierto, la Resolución Contran n ${ }^{\circ}$ 514/2014, que prevé la Política Nacional de Tránsito, tiene entre sus directrices en el Art. 5, Inciso III, alinea "c" fomentar el desarrollo de sistemas de transporte público y no motorizado.

Por último, está la política de aparcamiento que busca la sostenibilidad de la ciudad, donde hay que considerar el concepto de usuario preferido para seleccionar mejor la demanda, dada la limitada oferta de plazas.

\section{ESTUDIO DE CASO}

Los datos fueron recogidos junto a la Gerencia de Estadística do Departamento de Tránsito do Distrito Federal (Gerest-Detran/DF) para el período de 2000 a 2014. Los datos contienen la dirección donde ocurrió el accidente, la naturaleza (colisión, atropello, choque objeto fijo, queda del vehículo, etc.), la gravedad (mortales o heridos), fecha, hora, día de la semana y los tipos de vehículos implicados. 


\begin{tabular}{|c|c|c|c|c|c|c|c|c|c|c|c|c|c|c|c|c|}
\hline Severidad & Naturaleza & 2000 & 2001 & 2002 & 2003 & 2004 & 2005 & 2006 & 2007 & 2008 & 2009 & 2010 & 2011 & 2012 & 2013 & 2014 \\
\hline \multirow{9}{*}{ Herido } & Atropello de Animal & 0 & 1 & 1 & 0 & \begin{tabular}{|l|}
0 \\
\end{tabular} & 1 & 1 & 0 & 0 & \begin{tabular}{l|l}
0 \\
\end{tabular} & 0 & \begin{tabular}{|l|}
0 \\
\end{tabular} & 0 & 1 & 0 \\
\hline & Atropello de Peatone & 26 & 18 & 16 & 14 & 18 & 14 & 11 & 27 & 33 & 18 & 33 & 24 & 19 & 25 & 22 \\
\hline & Capotamento & 0 & 1 & 1 & 0 & 2 & 0 & 2 & 0 & 1 & 1 & 2 & 1 & 0 & 1 & 0 \\
\hline & Choque Objeto Fijo & 6 & 4 & 7 & 4 & 5 & 4 & 2 & 5 & 8 & 4 & 5 & 5 & 3 & 3 & 4 \\
\hline & Colisión & 37 & 30 & 24 & 21 & 34 & 29 & 26 & 50 & 54 & 60 & 60 & 62 & 43 & 52 & 53 \\
\hline & Otra & 0 & 0 & 0 & 1 & 1 & 1 & 2 & 1 & 0 & 1 & 1 & 1 & 0 & 0 & 0 \\
\hline & Queda de Persona & 1 & 1 & 2 & 3 & 2 & 2 & 0 & 1 & 1 & 2 & 4 & 8 & 3 & 4 & 6 \\
\hline & Queda del Vehículo & 3 & 1 & 0 & 2 & 0 & 1 & 2 & 3 & 0 & 6 & 5 & 7 & 2 & 5 & 2 \\
\hline & Tombamento & 0 & 0 & 0 & 0 & 0 & 0 & 1 & 0 & 0 & 1 & 0 & 0 & 0 & 0 & 0 \\
\hline \multirow{6}{*}{ Mortal } & Atropello de Peatone & 3 & 1 & 2 & 1 & 0 & 0 & 0 & 2 & 3 & 1 & 2 & 3 & 0 & 0 & 1 \\
\hline & Choque Objeto Fijo & 0 & 0 & 0 & 0 & 0 & 0 & 0 & 1 & 0 & 0 & 0 & 0 & 0 & 0 & 1 \\
\hline & Colisión & 2 & 0 & 1 & 0 & 0 & 0 & 1 & 2 & 3 & 1 & 0 & 0 & 1 & 3 & 0 \\
\hline & Queda de Persona & 0 & 0 & 1 & 0 & 0 & 0 & 0 & 0 & 0 & 0 & 0 & 0 & 0 & 0 & 0 \\
\hline & Queda del Vehículo & 0 & 0 & 0 & 0 & 0 & 0 & 0 & 0 & 0 & 1 & 1 & 1 & 0 & 0 & 0 \\
\hline & Total por año & 78 & 57 & 55 & 46 & 62 & 52 & 48 & 92 & 103 & 96 & 113 & 112 & 71 & 94 & 89 \\
\hline
\end{tabular}

Tabla 1 - Cantidad anual de accidentes por severidad y naturaleza

Los datos se dividieron en dos grupos. De 2000 a 2006, antes de la aplicación de los aparcamientos y de 2007-2014, para el período después de la construcción.

\begin{tabular}{|c|l|c|c|c|}
\hline Severidad & \multicolumn{1}{|c|}{ Naturaleza } & $\mathbf{2 0 0 0 - 2 0 0 6}$ & $\mathbf{2 0 0 7}-\mathbf{2 0 1 4}$ & Variación \\
\hline \multirow{5}{*}{ Herido } & Atropello de Animal & 0,6 & 0,1 & $-78 \%$ \\
\cline { 2 - 5 } & Atropello de Peatone & 16,7 & 25,1 & $50 \%$ \\
\cline { 2 - 5 } & Capotamento & 0,9 & 0,8 & $-13 \%$ \\
\cline { 2 - 5 } & Choque Objeto Fijo & 4,6 & 4,6 & $1 \%$ \\
\cline { 2 - 5 } & Colisión & 28,7 & 54,3 & $89 \%$ \\
\cline { 2 - 5 } & Otra & 0,7 & 0,5 & $-30 \%$ \\
\cline { 2 - 5 } & Queda de Persona & 1,6 & 3,6 & $131 \%$ \\
\cline { 2 - 5 } & Queda del Vehículo & 1,3 & 3,8 & $192 \%$ \\
\cline { 2 - 5 } & Tombamento & 0,1 & 0,1 & $-13 \%$ \\
\hline \multirow{5}{*}{ Mortal } & Média Heridos & 55,1 & 92,9 & $68 \%$ \\
\cline { 2 - 5 } & Atropello de Peatone & 1,0 & 1,5 & $50 \%$ \\
\cline { 2 - 5 } & Choque Objeto Fijo & 0,0 & 0,3 & - \\
\cline { 2 - 5 } & Quedisión & 0,6 & 1,3 & $119 \%$ \\
\cline { 2 - 5 } & Queda de Persona & 0,1 & 0,0 & $-100 \%$ \\
\hline & Média Fatales & 0,0 & 0,4 & - \\
\hline & Média General & 1,7 & 3,4 & $97 \%$ \\
\hline \multirow{2}{*}{} & & 56,9 & 96,3 & $69 \%$ \\
\hline
\end{tabular}

Tabla 2 - Resumen de media anual de accidentes por período y variación

En la Tabla 2 se observa que el número de heridos aumentó $68 \%$, debido principalmente al aumento de atropello de peatones y colisión. Queda de persona y queda del vehículo aumentaran en porcentaje. En cuanto a los accidentes mortales aumentaron 97\%, destacando el atropello de peatones y colisión. La queda de persona tiene prácticamente autobús como el vehículo envuelto (de los 41 casos, 40 auto carros y sólo un camión). A la queda del vehículo, de los 42 eventos, 4 eran con bicicletas, 1 con camioneta y 37 con motocicletas.

Como se ha mencionado, la dispersión de la travesía de peatones a lo largo de la vía y la aceleración/deceleración de vehículos en el rango de más alta velocidad de acceso al aparcamiento en el parterre central puede estar relacionado con el aumento en el número de accidentes fatales y heridos.

Fueron obtenidos del Sistema de Informação Georreferenciada do Departamento de Trânsito 
do Distrito Federal (Sig-Detran/DF) datos de 6 equipos de vigilancia electrónica (3 en cada sentido de la ruta), siendo 2 equipamientos de velocidad de tráfico (conocido como "pardal") y 4 reguladores electrónicos de velocidad (barreras electrónicas). Estos datos se refieren al período comprendido entre 2009 y 2014 sobre el volumen anual medio diario (VMDA), la cantidad media anual de violaciones diarias por exceso de velocidad y por consiguiente, el índice de infracciones (infracciones por volumen de vehículos).

En el local de mayor actividad (cuadra 101/201), cerca del acceso a la carretera, el VMDA era de 19.162 vehículos, con una tasa promedia de 8 infracciones diarias por cada 10.000 vehículos en el período de 2010 a 2014. El equipo ubicado en la cuadra 104 presentó VMDA de 9.811 vehículos y la tasa media de infracciones diarias de 6 infracciones por cada 10.000 vehículos en el período 2009 a 2014. No hubo cambios significativos en cualquiera de los equipos de vigilancia durante los años 2009-2014, sea por el número de vehículos, o sea por el número de infracciones.

Con relación a los accidentes con bicicletas, antes de la construcción de la ciclo vía (2000 a 2010) ocurrieron 13,9 accidentes por año, mientras que después de la ciclo vía (2011 a 2014) la tasa media fue de 9,0 accidentes por año. Esto permite considerar que la ciclo vía no fue la responsable por el elevado número de accidentes verificados a partir de 2007, sino implantación del aparcamiento.

\section{CONSIDERAÇÕES FINAIS}

Este estudio examinó el impacto en la seguridad vial de la obra de construcción de aparcamiento de vehículos en el parterre central de la Avenida Recanto das Emas. De las condiciones de implantación se verificó la ausencia de divisor físico con al menos $1 \mathrm{~m}$ de ancho (violación de la ley) y el conflicto al comienzo y al final del bolsillo de aparcamiento para la ciclo vía (implantada más tarde). El aumento en el número de accidentes mortales (97\%) y con lesiones (68\%) se llevó a cabo principalmente en relación con los atropellamientos de peatones y colisiones, con el entendimiento de que estas naturalezas de accidentes sufrieran influencia directa del aparcamiento en el parterre central en las condiciones que se implementó. La ausencia de una política de aparcamiento debe ser resaltada, así como una mejor evaluación de la funcionalidad y la jerarquía de la Avenida Recanto das Emas frente a la oferta y la colocación de plazas de aparcamiento. Consideraciones extraídas de estas análisis ayudaran a otros proyectos en el Distrito Federal, especialmente en la propuesta de aparcamiento en el parterre central de la avenida Paranoá.

\section{AGRADECIMIENTOS}

Los autores agradecen al Conselho Nacional de Desenvolvimento Científico e Tecnológico (CNPq), al Departamento de Trânsito do Distrito Federal y a la Escuela Superior Politécnica del Litoral.

\section{REFERENCIAS}


BRASIL (1997). Lei no 9.503, de 23 de setembro de 1997. Institui o Código de Trânsito Brasileiro. Diário Oficial da União, Brasília, DF. Disponível em: <http://www.planalto.gov.br/ccivil_03/leis/19503.htm>.

\section{COMISSÃO DE COORDENAÇÃO E DESENVOLVIMENTO REGIONAL DO NORTE}

(CCDR-N) (2008). Manual de planeamento das acessibilidades e da gestão viária. Volume 4. Princípios básicos de organização de redes viárias. Ministério do Ambiente e do Ordenamento do Território. Portugal.

CONSELHO NACIONAL DE TRÂNSITO (Brasil) (CONTRAN). Sinalização vertical de regulamentação / Contran-Denatran. 2a edição - Brasília : Contran, 2007. 220 p. : il. (Manual Brasileiro de Sinalização de Trânsito ; 1).

CONTRAN (2014). Resolução Contran no 514 (2014). Dispõe sobre a Política Nacional de Trânsito, seus fins e aplicação, e dá outras providências. Brasília, DF.

COMPANHIA DE PLANEJAMENTO DO DISTRITO FEDERAL (CODEPLAN). (2015). PDAD 2015: Recanto das Emas. Disponible en: <http://www.codeplan.df.gov.br/component/content/article/261-pesquisassocioeconomicas/319-pdad-2015.html>.

EMPRESA DE TRANSPORTES E TRÂNSITO DE BELO HORIZONTE (BHTRANS). (2010). Práticas de estacionamento em Belo Horizonte. Belo Horizonte, Minas Gerais. 